\title{
On the Validity of Some Approximations to the Appleton-Hartree Formula ${ }^{1}$
}

\author{
Kenneth Davies and G. A. M. King ${ }^{2}$
}

(December 9, 1960)

\begin{abstract}
The validity of some commonly used quasi-transverse and quasi-longitudinal approximations to the Appleton magneto-ionic formula is considered. Using the dipole approximation for the earth's magnetic field the various approximations for the refractive index are compared with the values computed from the complete formula for various geomagnetic latitudes and a frequency of 2.0 megacycles per second. It is found that certain approximations become very poor only a short distance from where they are exact and so care must be taken in their use. It is shown that a choice of two suitable approximations yields refractive indices of sufficient accuracy for all geomagnetic latitudes. Certain approximations to the group refractive indices are also considered.
\end{abstract}

\section{Introduction}

Approximations to the Appleton-Hartree formula have been considered by a number of workers [Booker, 1935; Rydbeck, 1940; Wostfold, 1951; Ratcliffe, 1959; Titheridge, 1959]. In most cases, however, the range of validity of the expressions used is difficult to assess. Although, with the advent of electronic computers, the necessity for using approximations in numerical work has decreased; there is still some need for them in practical work and also they are widely used to simplify theoretical discussions. We shall consider here the applicability of some approximate formulas for the case of the earth's dipole magnetic field and for frequencies normally used in ionospheric sounding at vertical incidence. Calculations involving the time of flight of radio pulses require the group refractive index and so we have included a consideration of some approximations to the formula for this quantity. (The effect of electronic collisions on the phase and group indices has been neglected.)

\section{Definitions}

$N=$ electron density

$f=$ frequency

$f_{H}=$ gyrofrequency

$f_{N}=$ plasmafrequency

$\theta=$ angle between direction of propagation and the magnetic field

$x=\left(\frac{f_{N}}{f}\right)^{2}$

$y=\frac{f_{H}}{f}$

$\xi^{2}=f^{2}-f f_{H}$

1 Contribution from Central Radio Propagation Laboratory, National Bureau of Standards, Boulder, Colo.

2 Present address, Geophysical Observatory, P.O. Box 2111, Christchurch, New 2 sent and

\section{Approximations}

The expression for the phase refractive index in the ordinary mode, $n_{+}$, is

$$
\frac{x}{1-n_{+}^{2}}=1-\frac{y^{2} \sin ^{2} \theta}{2(1-x)}+\sqrt{\frac{y^{4} \sin ^{4} \theta}{4(1-x)^{2}}+y^{2} \cos ^{2} \theta} \cdot
$$

The quasi-transverse approximations, $n_{1}$ and $n_{2}$, are given by

$$
\begin{aligned}
& \frac{x}{1-n_{1}^{2}}=1, \\
& \frac{x}{1-n_{2}^{2}}=1+(1-x) \cot ^{2} \theta,
\end{aligned}
$$

and the quasi-longitudinal approximations are given by $n_{3}, n_{4}$, and $n_{5}$

$$
\begin{aligned}
& \frac{x}{1-n_{3}^{2}}=1+|y \cos \theta|, \\
& \frac{x}{1-n_{4}^{2}}=1+|y \cos \theta|-\frac{y^{2} \sin ^{2} \theta}{2(1-x)}, \\
& \frac{x}{1-n_{5}^{2}}=1+y .
\end{aligned}
$$

The expression for the phase refractive index in the extraordinary mode, $n_{-}$, is

$$
\frac{x}{1-n_{-}^{2}}=1-\frac{y^{2} \sin ^{2} \theta}{2(1-x)}-\sqrt{\frac{y^{4} \sin ^{4} \theta}{4(1-x)^{2}}+y^{2} \cos ^{2} \theta} .
$$

The quasi-transverse approximations are given by $n_{8}$, $n_{9}$, and $n_{11}$ and the quasi-longitudinal approximations are given by $n_{6}, n_{7}$, and $n_{10}$.

$$
\frac{x}{1-n_{6}^{2}}=1-y,
$$




$$
\begin{aligned}
& \frac{x}{1-n_{7}^{2}}=1-|y \cos \theta|, \\
& \frac{x}{1-n_{8}^{2}}=1-\frac{y^{2} \sin ^{2} \theta}{1-x}, \\
& \frac{x}{1-n_{9}^{2}}=1-\frac{y^{2}}{1-x}, \\
& \frac{x}{1-n_{10}^{2}}=1-|y \cos \theta|-\frac{y^{2} \sin ^{2} \theta}{2(1-x)}, \\
& \frac{x}{1-n_{11}^{2}}=1-\frac{y^{2} \sin ^{2} \theta}{(1-x)}-(1-x) \cot ^{2} \theta .
\end{aligned}
$$

The group refractive index $\left(n^{\prime}=n+f \frac{d n}{d f}\right)$ is given by the lengthy formula,

$$
\begin{aligned}
\left(n_{ \pm}\right)^{\prime}= & \left(n_{ \pm}\right)+\left\{1-\left(n_{ \pm}\right)^{2}\right\}^{2}\left\{\frac{1}{\left(n_{ \pm}\right) x}+\frac{y^{2} \sin ^{2} \theta}{2\left(n_{ \pm}\right)(1-x)^{2}}\right. \\
& \left. \pm \frac{2(1-x)^{3} y^{2} \cos ^{2} \theta-x y^{4} \sin ^{4} \theta}{2\left(n_{ \pm}\right) x(1-x)^{2} \sqrt{y^{4} \sin ^{4} \theta+4 y^{2}(1-x)^{2} \cos ^{2} \theta}}\right\},
\end{aligned}
$$

and we discuss these approximations to it:

Ordinary Mode, Quasi-transverse Approximation

$$
\begin{aligned}
\left(n_{2}\right)^{\prime}= & \left(n_{2}\right) \\
& +\frac{x\left(1+\cot ^{2} \theta\right)}{\left(n_{2}\right)\left(1+\cot ^{2} \theta\right)\left\{1+(1-2 x) \cot ^{2} \theta\right\}+\left(n_{2}\right) x \cot ^{4} \theta}
\end{aligned}
$$

Extraordinary Mode, Quasi-longitudinal Approximation

$$
\left(n_{6}\right)^{\prime}=\left(n_{6}\right)+\frac{2 x-x y}{2\left(n_{6}\right)(1-y)^{2}}
$$

Extraordinary Mode, Quasi-transverse Approximation

$$
\left(n_{9}\right)^{\prime}=\left(n_{\ni}\right)+\frac{x(1-x)^{2}+x^{2} y^{2}}{\left(n_{9}\right)\left(1-x-y^{2}\right)^{2}}
$$

\section{Discussion}

For a dipole field, the propagation angle, $\theta$, and the geomagnetic latitude, $\lambda$, are related for vertical incidence propagation by

$$
\cot \theta=2 \tan \lambda . \quad \text { (See table } 1)
$$

Therefore, the discussion will be carried on in terms of $\lambda$.

(a) Phase index in the ordinary mode. In figures $1 \mathrm{a}, \mathrm{b}, \mathrm{c}, \mathrm{d}, \mathrm{e}$, and $\mathrm{f}$ we have plotted the variations of $n_{+}$and the approximations $n_{1}$ to $n_{5}$ as functions of geomagnetic latitude (the propagation angle, $\theta$, is also indicated). The graphs are for a frequency, $f$, of $2 \mathrm{Mc} / \mathrm{s}$, and each one treats a different plasmafrequency between $0.5 f$ and $0.998 f$. We also exam- ined the cases of $f=4 \mathrm{Mc} / \mathrm{s}$ and $f=8 \mathrm{Mc} / \mathrm{s}$, and found that all the approximations held more closely than at $2 \mathrm{Mc} / \mathrm{s}$, but they maintained their relative order.

The best approximation to $n_{+}$appears to be a combination of $n_{2}$ and $n_{4}$. At lower latitudes $n_{2}$ is the more accurate with a positive error, and at higher latitudes $n_{4}$ is the more accurate with a negative error. The latitudes at which these errors are equal in magnitude together with the percent errors are given in table 2 . It is of interest to note that, where the errors are largest $\left(f_{N} / f=0.998\right)$ they only apply to a very narrow range of $\lambda$. Figures $2 \mathrm{a}$ and $2 \mathrm{~b}$ which show $n$ as a function of plasma frequency for $f=2.00 \mathrm{Mc} / \mathrm{s}$ and latitudes of $60^{\circ}$ and $30^{\circ}$ respec-

\begin{tabular}{|c|c|c|}
\hline$x^{\circ}$ & $\theta$ & $f_{H}$ \\
\hline $\begin{array}{r}0 \\
5 \\
10 \\
15 \\
20\end{array}$ & $\begin{array}{ll}90^{\circ} & 00 \\
80^{\circ} & 04 \\
70^{\circ} & 35 \\
61^{\circ} & 49 \\
54^{\circ} & 57\end{array}$ & $\begin{array}{c}M c / s \\
0.8000 \\
.8080 \\
.8354 \\
.8763 \\
.9295\end{array}$ \\
\hline $\begin{array}{l}25 \\
30 \\
35 \\
40 \\
45\end{array}$ & $\begin{array}{ll}47^{\circ} & 00 \\
40^{\circ} & 54 \\
35^{\circ} & 32 \\
30^{\circ} & 47 \\
26^{\circ} & 34\end{array}$ & $\begin{array}{r}.9895 \\
1.0583 \\
1.1266 \\
1.1974 \\
1.2649\end{array}$ \\
\hline $\begin{array}{l}50 \\
55 \\
60 \\
65 \\
70\end{array}$ & $\begin{array}{ll}22^{\circ} & 46 \\
19^{\circ} & 19 \\
16^{\circ} & 06 \\
14^{\circ} & 08 \\
10^{\circ} & 19\end{array}$ & $\begin{array}{l}\text { 1. } 3290 \\
\text { 1. } 3888 \\
\text { 1. } 4422 \\
\text { 1. } 4890 \\
1.5284\end{array}$ \\
\hline $\begin{array}{l}75 \\
80 \\
85 \\
90\end{array}$ & $\begin{array}{ll}07^{\circ} & 38 \\
05^{\circ} & 02 \\
02^{\circ} & 30 \\
00^{\circ} & 00\end{array}$ & $\begin{array}{l}1.5594 \\
1.5819 \\
1.5952 \\
1.6000\end{array}$ \\
\hline
\end{tabular}
tively confirm the above conclusions. Table 3 gives the latitude ranges over which the various

\begin{tabular}{|c|c|c|}
\hline$\frac{f_{N}}{f}$ & $\lambda$ & Error \\
\hline $\begin{array}{r}0.500 \\
.600 \\
.700 \\
.800 \\
.900\end{array}$ & $\begin{array}{c}\text { deg } \\
12 \\
13 \\
15 \\
22 \\
33\end{array}$ & $\begin{array}{l}\% \\
0.6 \\
.8 \\
1.5 \\
2.7 \\
7.8\end{array}$ \\
\hline $\begin{array}{l}.930 \\
.960 \\
.980 \\
.990 \\
.995\end{array}$ & $\begin{array}{l}41 \\
52 \\
62 \\
73 \\
76\end{array}$ & $\begin{array}{r}9.1 \\
11.7 \\
11.8 \\
12.0 \\
14.7\end{array}$ \\
\hline .998 & 82 & 8.5 \\
\hline
\end{tabular}
approximations are within 5 percent of the true value.

The most commonly used approximation in the ordinary mode is $n_{3}$. It is interesting to note that it holds well only at very high latitudes and, almost fortuitously, at the equator. (One should not use

TABLE 1. Variation of propagation angle, $\theta$, and gyrofrequency with geomagnetic latitude for a dipole field

$\cot 9=2 \tan \lambda$

TABLE 2. Point of equal error in $\mathrm{n}_{2}$ and $\mathrm{n}_{4}$ from $\mathrm{n}_{+}$

For 0.998 , the data are insufficient to accurately plot $n_{4}$; the figures shown are the minimum values. 
TABLE 3. Areas of better than 5 percent accuracy in approximations to $\mathrm{n}_{+}$

$\lambda=$ Geomagnetic latitude (deg)

\begin{tabular}{|c|c|c|c|c|c|}
\hline$\underline{f_{N}}$ & $n_{1}$ & $n_{2}$ & $n_{3}$ & $n_{4}$ & $n_{5}$ \\
\hline & $\lambda \overline{<}_{41}$ & $+\underset{37}{+}$ & $\stackrel{+}{+}<00$ & $0<\bar{\lambda}<0$ & $\stackrel{+}{+}<00$ \\
\hline $\begin{array}{r}0.500 \\
.600\end{array}$ & $\begin{array}{l}\hat{\lambda}<25 \\
<<25\end{array}$ & $\lambda<30$ & $\begin{array}{l}0 \leqq \lambda \leqq 90 \\
0 \leqq \lambda \leqq 90\end{array}$ & $\begin{array}{l}0 \leqq \lambda \equiv 90 \\
0 \leqq \lambda \equiv 90\end{array}$ & $\begin{array}{c}0 \leqq \lambda \triangleq 90 \\
13<\lambda\end{array}$ \\
\hline .700 & $\lambda<19$ & $\lambda<27$ & $0 \leqq \lambda \leqq 90$ & $04<\lambda$ & $27<\lambda$ \\
\hline .800 & $\lambda<13$ & $\lambda<30$ & $30<\lambda$ & $16<\lambda$ & $41<\lambda$ \\
\hline .900 & $\lambda<12$ & $\lambda<32$ & $\begin{aligned} & 52<\lambda \\
& \quad \lambda<03\end{aligned}$ & $35<\lambda$ & $55<\lambda$ \\
\hline .930 & $\lambda<12$ & $\lambda<34$ & $61<\lambda$ & $45<\lambda$ & $63<\lambda$ \\
\hline .960 & $\lambda<12$ & $\lambda<43$ & $69<\lambda$ & $56<\lambda$ & $70<\lambda$ \\
\hline .980 & $\lambda<14$ & $\lambda<50$ & $77<\lambda$ & $66<\lambda$ & $77<\lambda$ \\
\hline .990 & $\lambda<15$ & $\lambda<66$ & $82<\lambda$ & $75<\lambda$ & $82<\lambda$ \\
\hline .995 & $\lambda<18$ & $\lambda<71$ & $\begin{aligned} 86<\lambda & \\
\lambda & =0\end{aligned}$ & $82<\lambda$ & $86<\lambda$ \\
\hline .998 & $\lambda<22$ & $\lambda<80$ & $\begin{array}{r}88<\lambda \\
\quad \lambda=0\end{array}$ & $84<\lambda$ & $88<\lambda$ \\
\hline
\end{tabular}

$n_{3}$ near the equator if there is the slightest doubt that the propagation angle is exactly zero.) In the latitude range where $n_{3}$ is satisfactory, the slightly simpler formula, $n_{5}$, is almost as good.

(b) Group index in the ordinary mode. The group refractive index, $n^{\prime}$, is given by

$$
n^{\prime}=n+f \frac{d n}{d f} .
$$

This equation can be applied to the approximate formulas for $n$ to give expressions for $n^{\prime}$. Clearly, it is not worth doing this for $n_{3}$ and $n_{5}$ since these are poor approximations to start with. Also $n_{4}^{\prime}$ is almost as complicated as $n_{+}^{\prime}$, and is, therefore, of little interest. So, figures $3 \mathrm{a}, \mathrm{b}, \mathrm{c}, \mathrm{d}, \mathrm{e}$, and $\mathrm{f}$ give a comparison of only $n_{+}^{\prime}$ and $n_{2}^{\prime}$.

As the region making the greatest contribution to the group path is where $f_{N}$ is almost equal to $f$, it seems wise to restrict the use of $n_{2}^{\prime}$ to latitudes less than $\lambda=30^{\circ}$.

(c) Phase index in the extraordinary mode. Curves showing the variations with latitude of $n_{-}$and the approximations $n_{6}$ to $n_{11}$ are given in figures $4 \mathrm{a}, \mathrm{b}$, $c, d$, e, and $\mathrm{f}$; the latitude ranges over which the various approximations hold to within 5 percent are set out in table 4.

$n_{10}$ is the extraordinary analogue of $n_{4}$ and $n_{11}$ is the analogue of $n_{2} . \quad n_{10}$ and $n_{11}$ are satisfactory only at high and at low latitudes respectively for small values of $f_{N} / \xi$. A combination of $n_{10}$ and $n_{11}$ is not satisfactory for values of $f_{N} / \xi$ approaching unity as is the case for $n_{4}$ and $n_{2}$ in the ordinary mode. For larger values of $f_{N} / \xi$, the choice seems to lie between $n_{6}$ at high latitudes and $n_{9}$ at low latitudes. It appears that approximations in the extraordinary mode are not really satisfactory at low-middle latitudes $\left(10^{\circ}\right.$ to $\left.30^{\circ}\right)$.

The approximation most commonly used for the extraordinary mode, $n_{7}$, is noticeably poor except at the pole (and at very low values of $f_{N} / \xi$ ). A discussion of $n_{6}$ and $n_{7}$ has been given by King [1960].
TABLE 4. Areas of closer than 5 percent accuracy in approximations to $\mathrm{n}$ -

$\lambda=$ Geomagnetic latitude (deg)

\begin{tabular}{|c|c|c|c|c|c|}
\hline$\frac{f_{N}}{\xi}$ & $n_{6}$ & $n_{7}$ & $n_{8}$ & $n_{9}$ & $n_{10}$ \\
\hline $\begin{array}{r}0.500 \\
.600 \\
.700 \\
.800\end{array}$ & $\begin{array}{l}0 \leqq \lambda \leqq 90 \\
12 \leqq \lambda \\
23<\lambda \\
31<\lambda\end{array}$ & $\begin{array}{c}0 \leqq \lambda \leqq 90 \\
0 \leqq \lambda \leqq 90 \\
45 \lesseqgtr \lambda \\
61<\lambda\end{array}$ & $\begin{array}{l}\lambda<29 \\
\lambda<19 \\
\lambda<14 \\
\lambda<08\end{array}$ & $\begin{array}{l}\lambda<56 \\
\lambda<19\end{array}$ & $\begin{array}{c}0 \leqq \lambda \leqq 90 \\
0 \leqq \lambda \leqq 90 \\
0 \leqq \lambda \leqq 90 \\
08<\lambda\end{array}$ \\
\hline $\begin{array}{l}.900 \\
.930 \\
.960 \\
.980 \\
.990\end{array}$ & $\begin{array}{l}38<\lambda \\
38<\lambda \\
40<\lambda \\
42<\lambda \\
38<\lambda\end{array}$ & $\begin{array}{l}75<\lambda \\
79<\lambda \\
83<\lambda \\
86<\lambda \\
87<\lambda\end{array}$ & $\begin{array}{l}\lambda<06 \\
\lambda<04 \\
\lambda<01 \\
\lambda<01 \\
\lambda<01\end{array}$ & $\begin{array}{l}\lambda<12 \\
\lambda<08 \\
\lambda<10\end{array}$ & $\begin{array}{l}23>\lambda \\
31<\lambda \\
41<\lambda \\
48<\lambda \\
55<\lambda\end{array}$ \\
\hline $\begin{array}{l}.995 \\
.998\end{array}$ & $\begin{array}{l}40<\lambda \\
37<\lambda\end{array}$ & $\begin{array}{l}88<\lambda \\
89<\lambda\end{array}$ & $\begin{array}{l}\lambda<01 \\
\lambda<01\end{array}$ & $\lambda<10$ & $\begin{array}{l}61<\lambda \\
66<\lambda\end{array}$ \\
\hline
\end{tabular}

Likewise, $n_{8}$ is good only at the equator where it degenerates into $n_{9}$, a simpler formula.

Plots of $n$ versus $f_{N} / \xi$ are given in figures $5 \mathrm{a}$ and $5 \mathrm{~b}$ so that the above discussion can be considered in another form.

(d) Group index in the extraordinary mode. Figures $6 \mathrm{a}, \mathrm{b}, \mathrm{c}, \mathrm{d}, \mathrm{e}$, and $\mathrm{f}$ compares $n_{-}^{\prime}, n_{6}^{\prime}$, and $n_{9}^{\prime}$. $n_{6}^{\prime}$ is surprisingly good at least for latitudes above $45^{\circ}$. $\quad n_{9}^{\prime}$ holds exactly at the equator, but its use elsewhere is questionable; of course, at the equator, $n_{9}^{\prime}$ degenerates into the transverse case.

\section{Conclusions}

Two formulas $n_{4}$ and $n_{2}$ are preferred for the phase index in the ordinary mode at high and low latitudes respectively. The latter gives rise to a suitable formula for the group index at low latitudes.

The phase refractive index in the extraordinary mode is not so easily treated. However, here again two formulas, $n_{6}$ and $n_{9}$, are probably adequate for many purposes. The most serious difficulties occur at low-middle latitudes. $n_{6}$ gives rise to a suitable formula for the group index at high latitudes.

We wish to point out that there is a tendency in the literature to stress certain approximations; e.g., $n_{1}, n_{3}$, and $n_{7}$, and to use them outside their proper range of applicability.

The authors acknowledge the help of Irving Littman who carried out many of the computations involved.

\section{References}

Booker, H. G., The application of the magneto-ionic theory to the ionosphere, Proc. Roy. Soc. 150, 267 (1935).

King, G. A. M., Use of logarithmic frequency spacing in ionogram analysis, J. Research NBS 64D, 501 (1960).

Ratcliffe, J. A., The magneto-ionic theory and its applications to the ionosphere (Cambridge Univ. Press, London, 1959).

Rydbeck, O. E. H., On the propagation of electro-magnetic waves in an ionized medium and the calculation of the heights of the ionized layers of the atmosphere, Pbil. Mag. 30, 282 (1940).

Titheridge, J. E., Ray paths in the ionosphere, J. Atmospheric and Terrest. Phys. 14, 50 (1959).

Westfold, K. C., The interpretation of the magneto-ionic theory, J. Atmospheric and Terrest. Phys. 1, 152 (1951). 

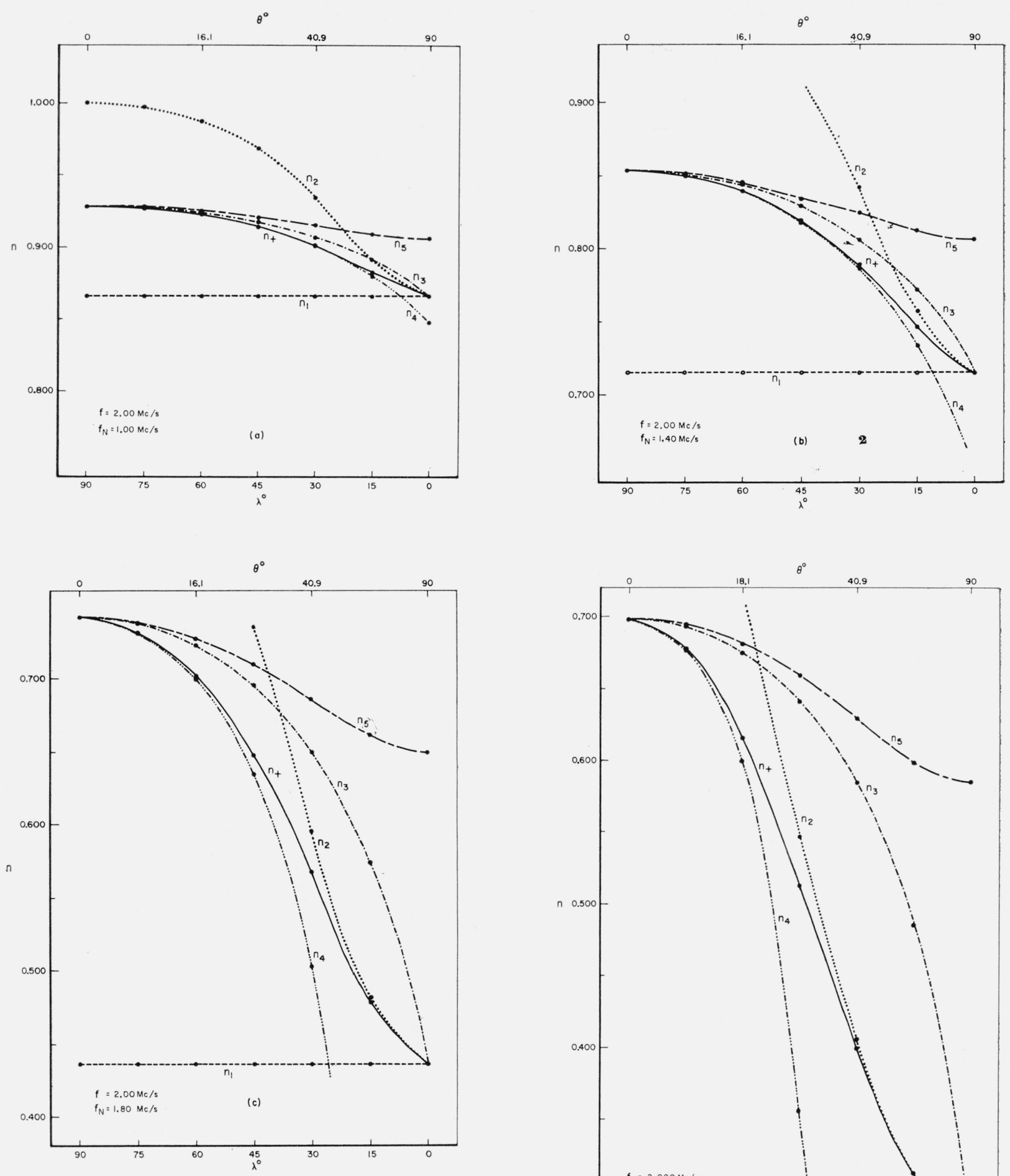

FIgURE 1. Comparisons of the geomagnetic latitude variation of the phase refractive index in the ordinary mode $\left(\mathrm{n}_{+}\right)$and various approximations to it.

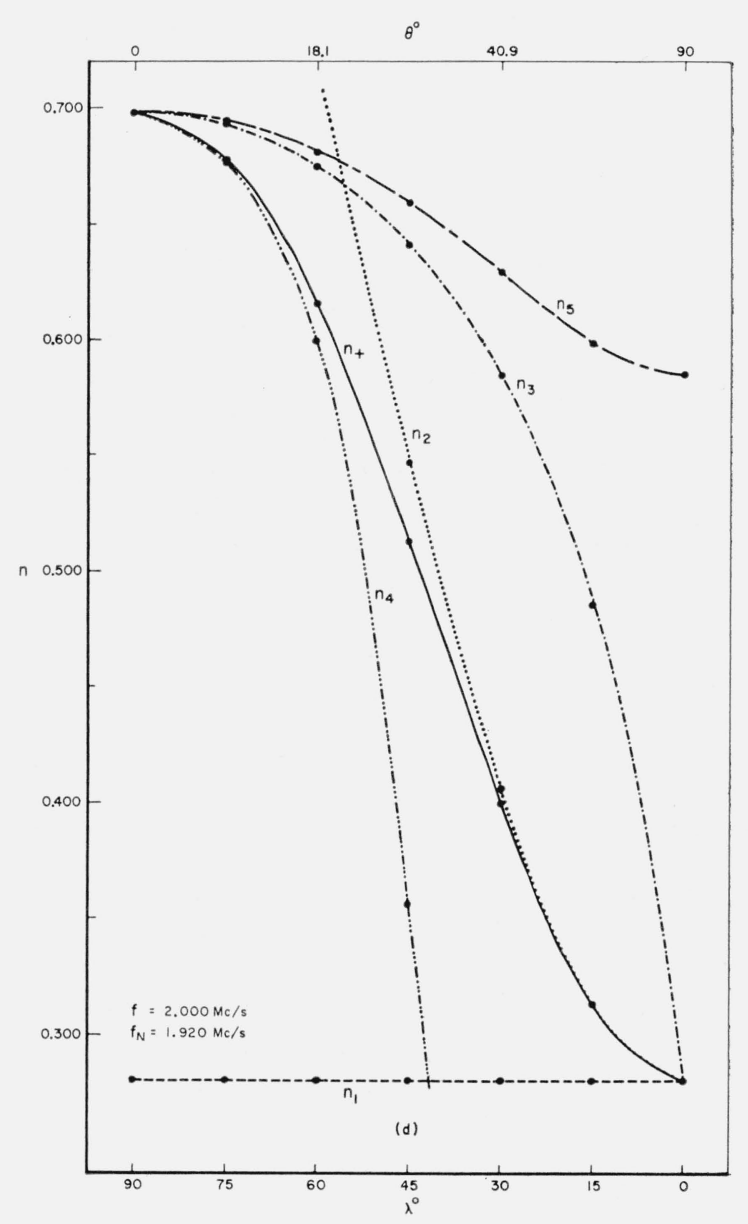



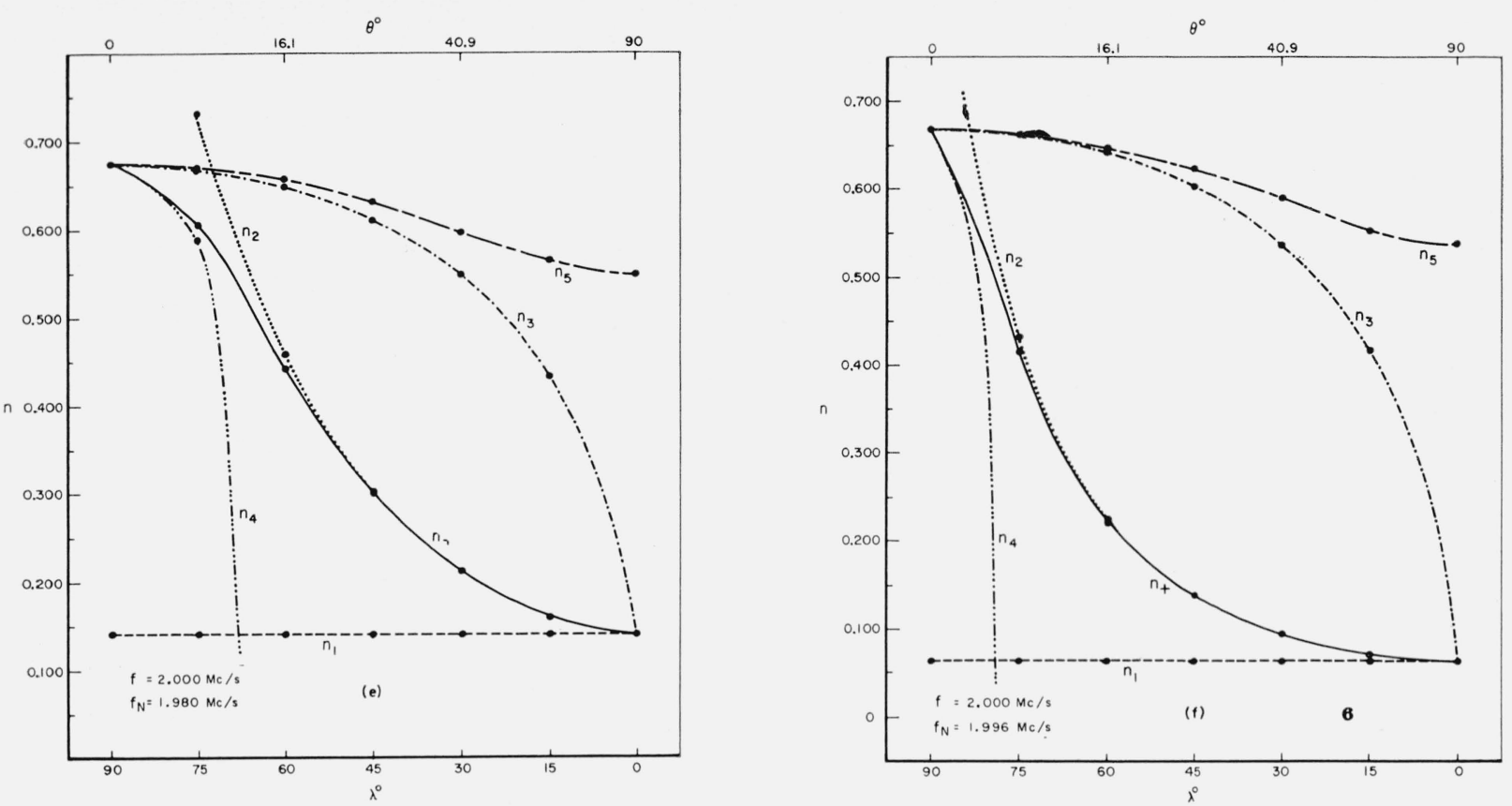

FIGURE 1. Comparisons of the geomagnetic latitude variation of the phase refractive index in the ordinary mode $\left(\mathrm{n}_{+}\right)$and various approximations to it-Continued
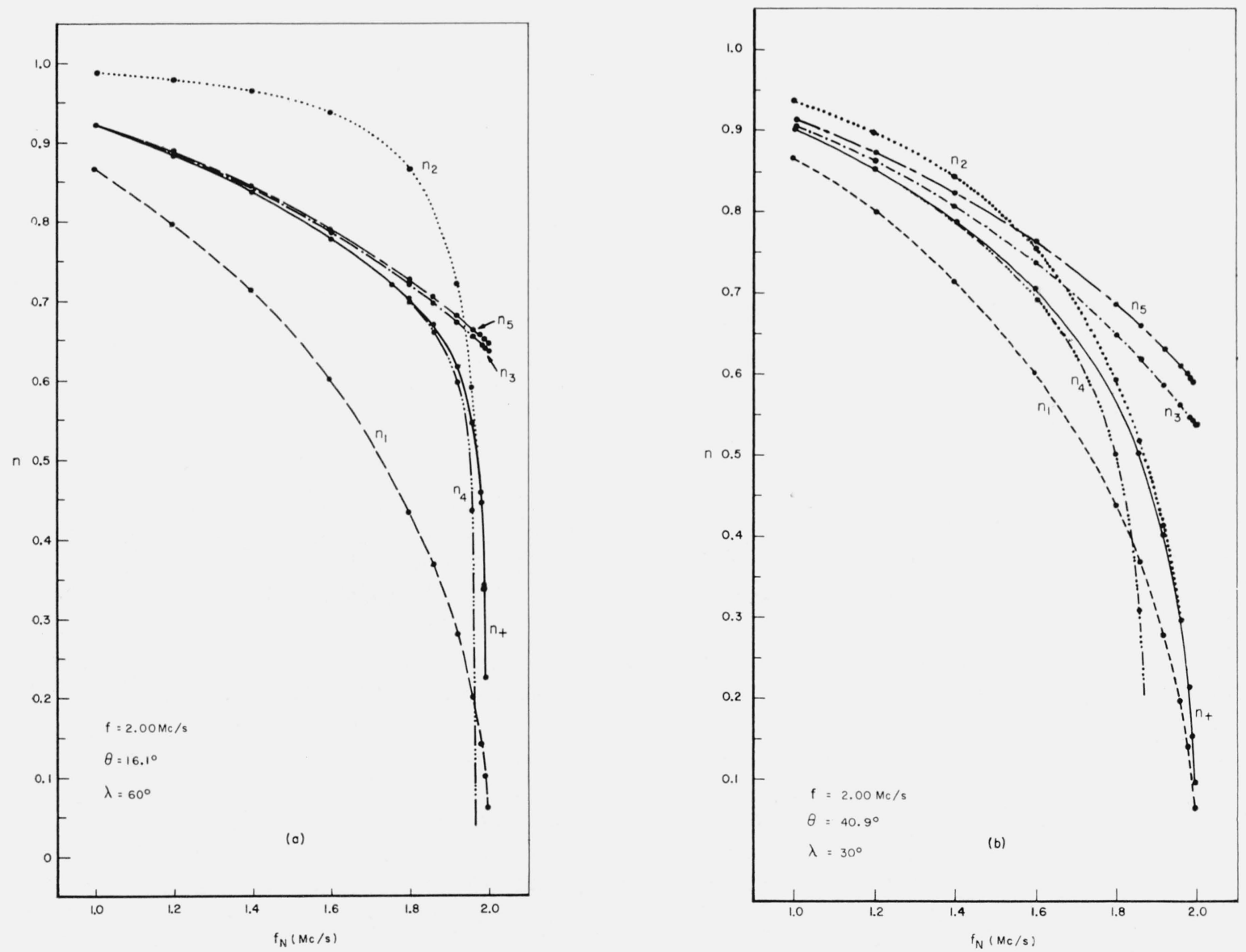

FIGURE 2. Comparisons of the variations, with plasma frequency, of the phase refractive index in the ordinary mode ( $n_{+}$) various approximations to it at geomagnetic latitudes of $60^{\circ}$ and $30^{\circ}$. 

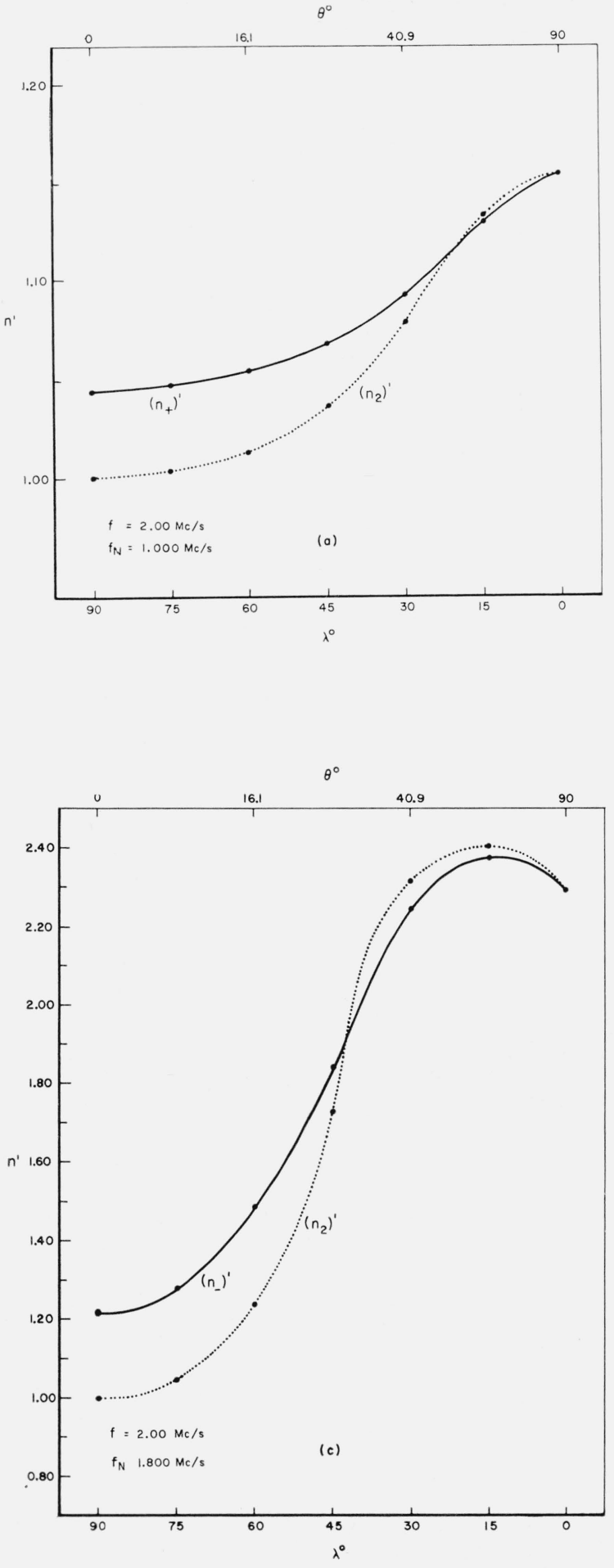

FIGURE 3. The latitudinal variation of the group refractive index in the ordinary mode and an approximation to it.
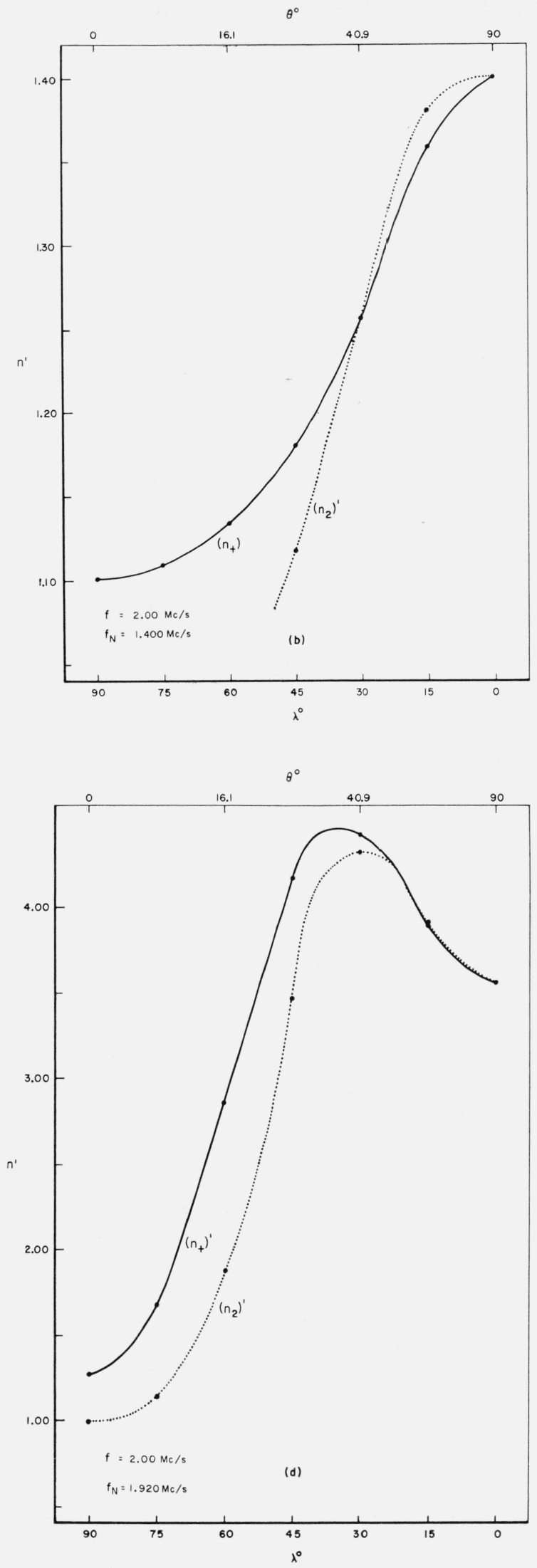

328 


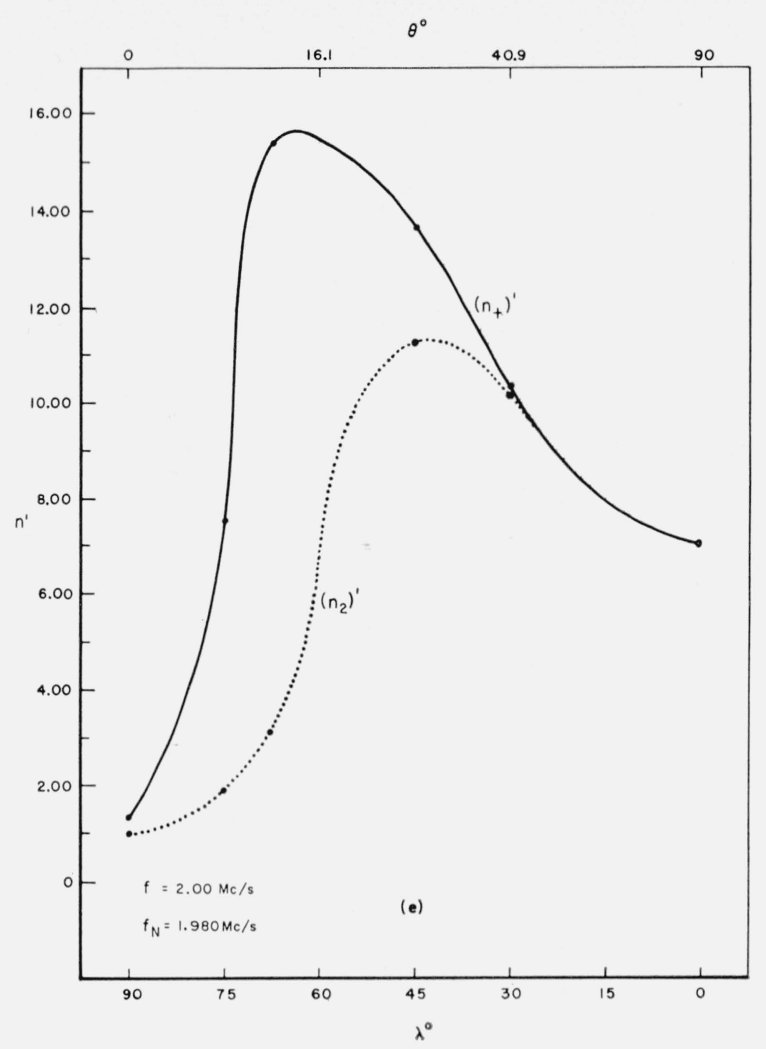

FIGURE 3. The latitudinal variation of the group refractive index in the ordinary mode and an approximation to it-Continued.

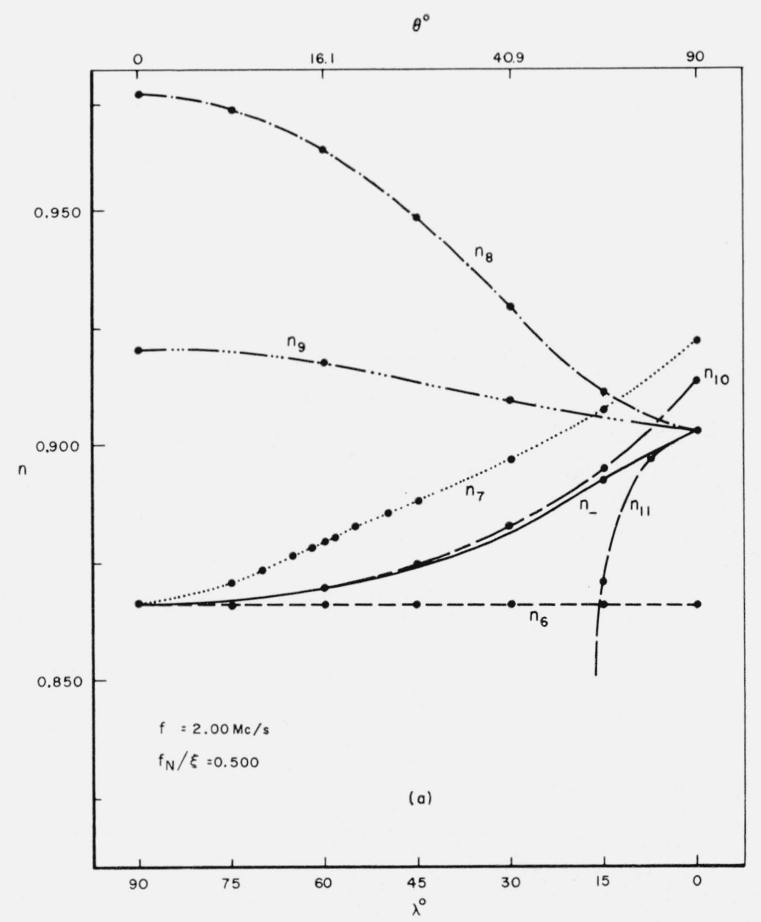

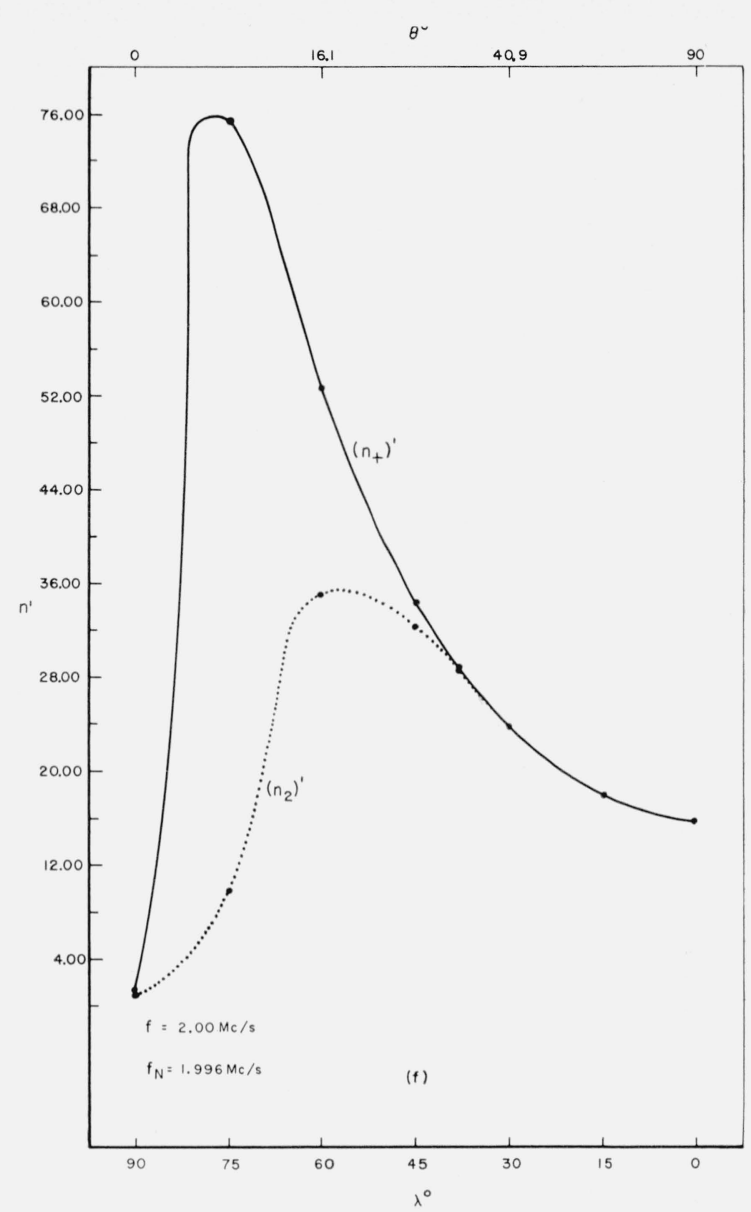

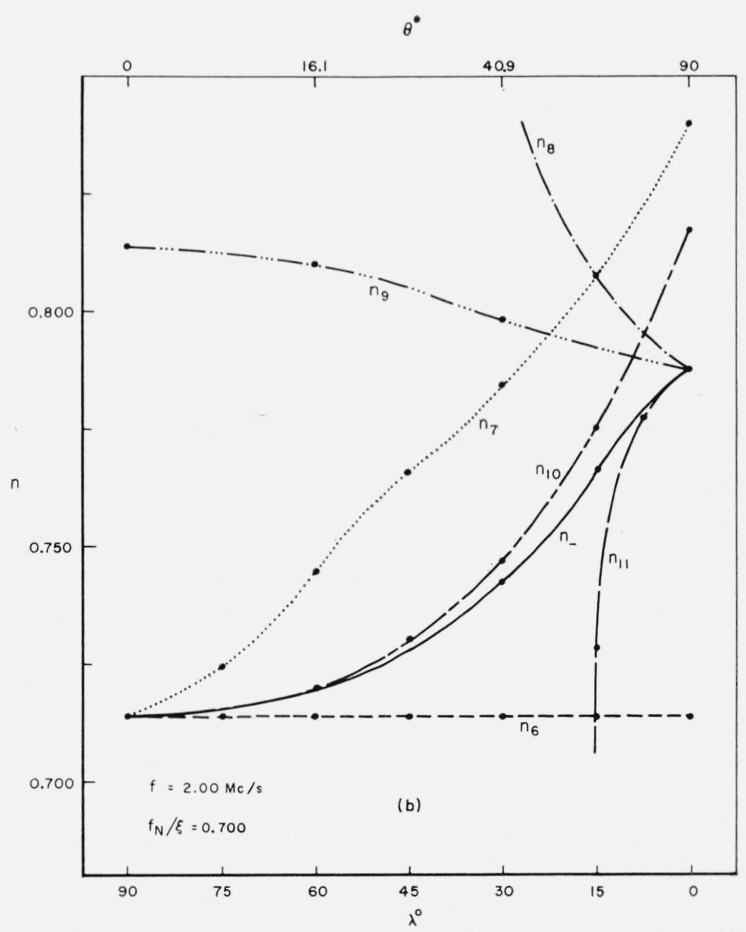

Figure 4. Comparisons of the geomagnetic latitude variations of the phase refractive index in the extraordinary mode ( $\mathrm{n}_{-}$) and various approximations to it. 

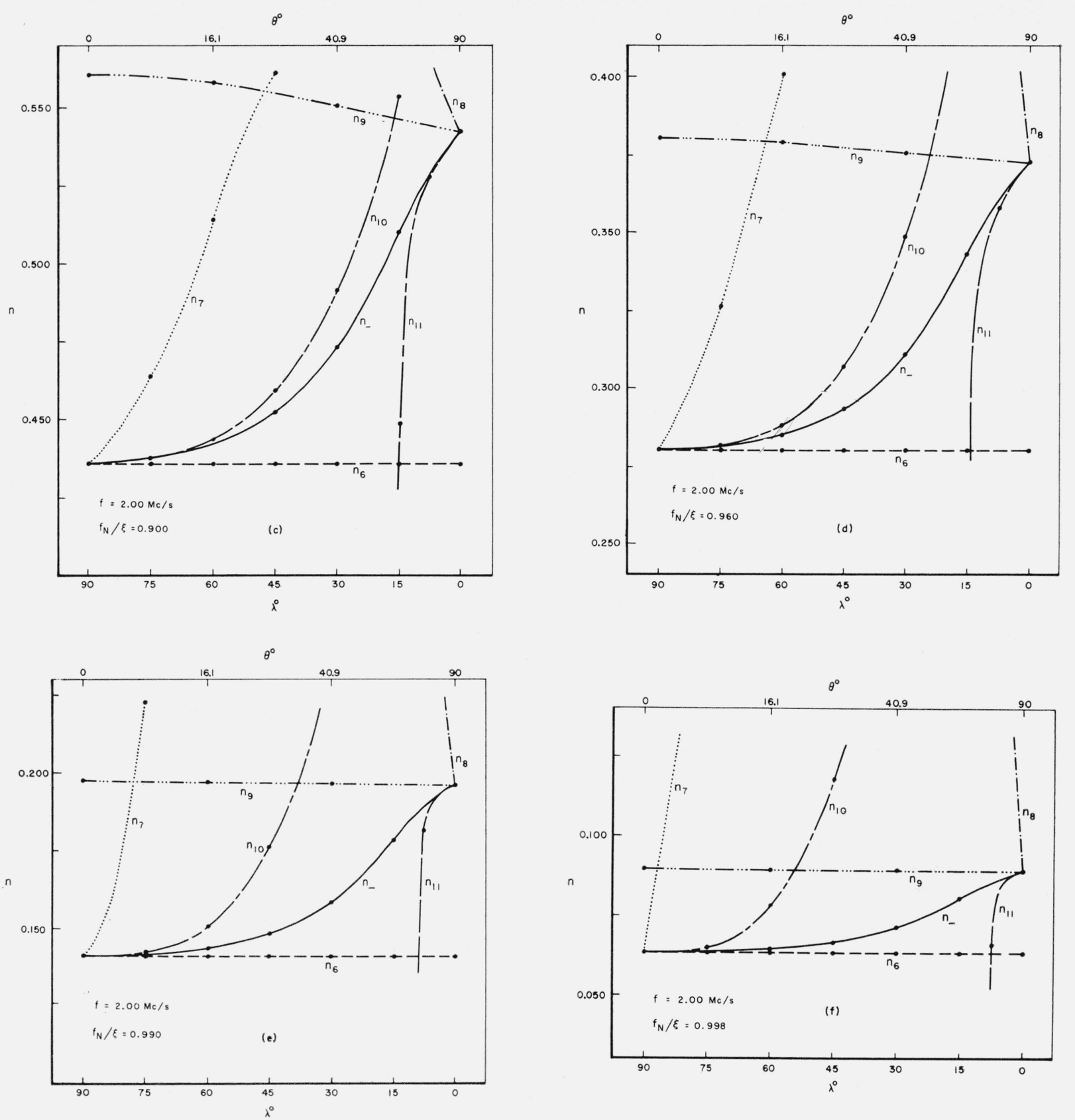

FIGURE 4. Comparisons of the geomagnetic latitude variations of the phase refractive index in the extraordinary mode ( $\mathrm{n}_{-}$) and various approximations to it-Continued 

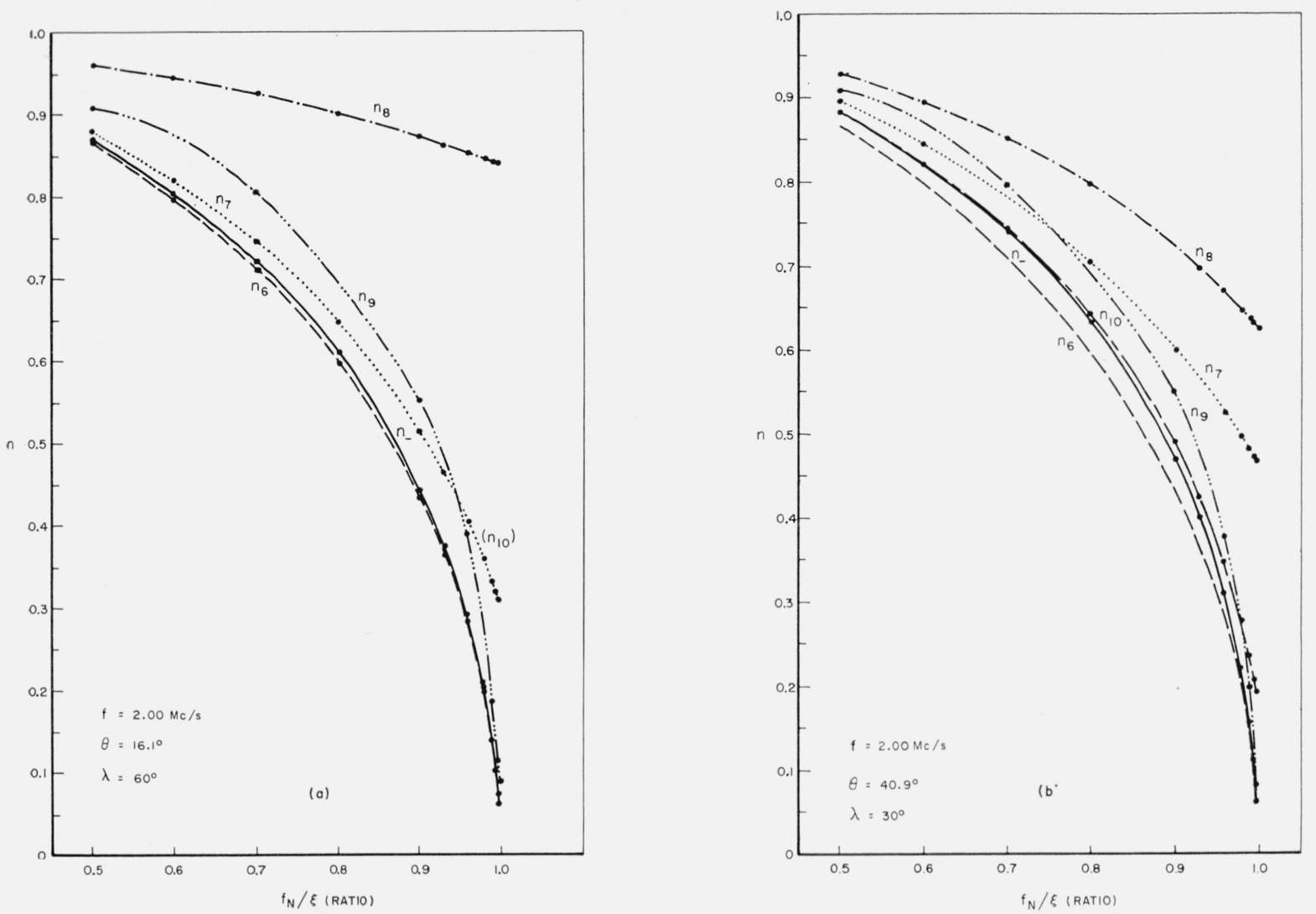

FIGURE 5. Comparisons of the variations, with the normalized plasmafrequency, of the phase refractive index in the extraordinary mode $\left(\mathrm{n}_{-}\right)$and various approximations to it at geomagnetic latitudes of $60^{\circ}$ and $30^{\circ}$.

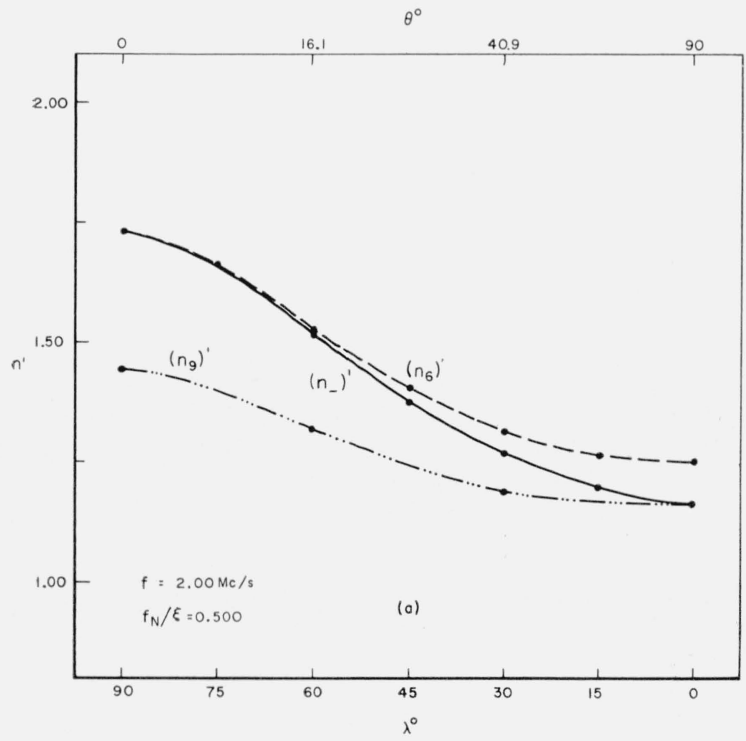

FIGURE 6. The latitudinal variation of the group refractive index in the extraordinary mode and two approximations to it.

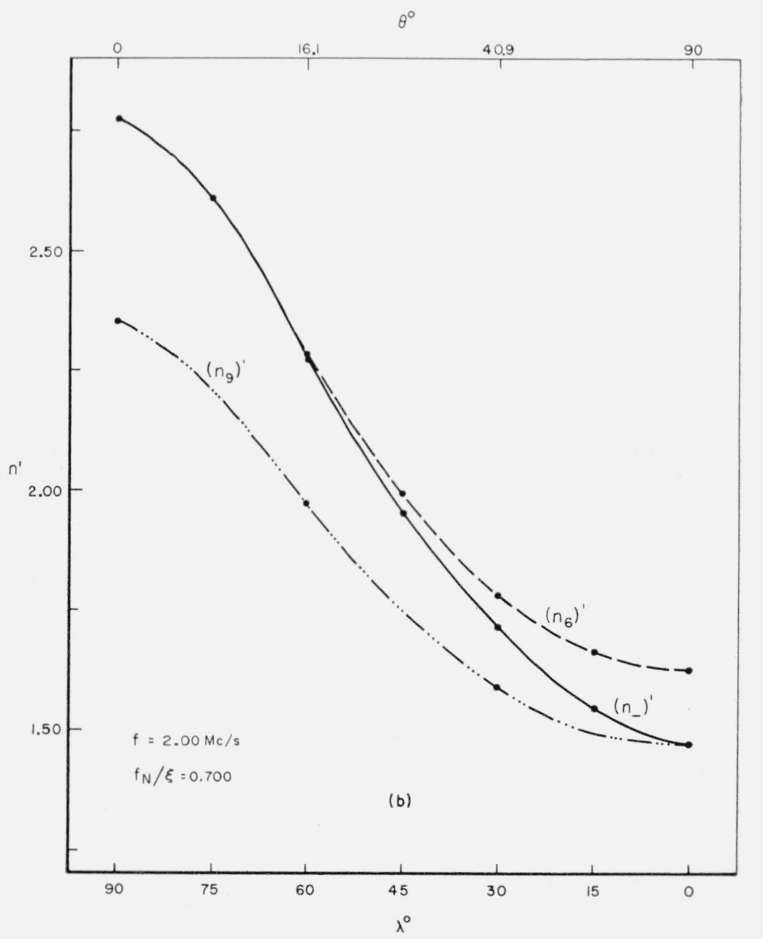



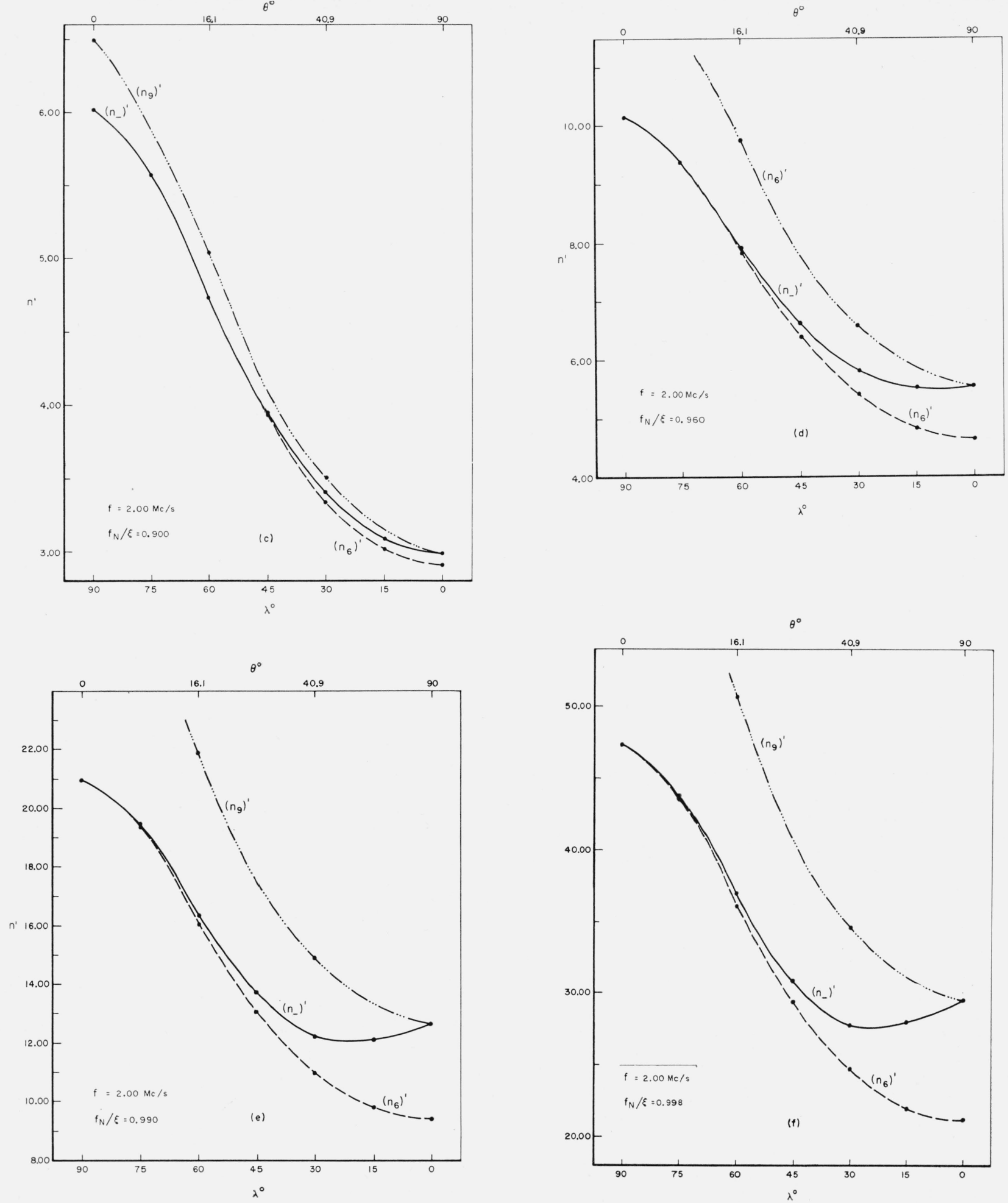

FIGURE 6. The latitudinal variation of the group refractive index in the extraordinary mode and two approximations to it -Con.

(Paper 65D4-136)

332 